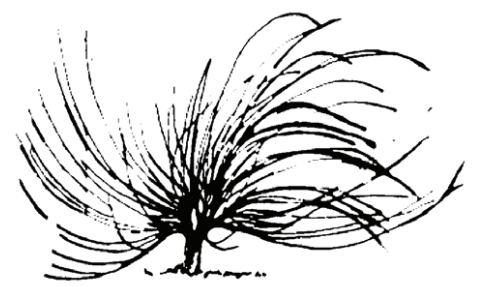

\title{
EI Trabajo Colaborativo en la Constitución de Equipos de Trabajo: La Experiencia del Proyecto Perfiles, Dinámicas y Desafíos de la Educación Costarricense
}

\author{
Karolina Campos Jiménez ${ }^{1}$ \\ Universidad Nacional \\ Heredia, Costa Rica \\ kcamposjmnz@gmail.com
}

\author{
Vivian Carvajal Jiménez $z^{2}$ \\ Universidad Naciona \\ Heredia, Costa Rica \\ vivian.carvajal@gmail.com
}

Susana Murillo León
Universidad Nacional
Heredia, Costa Rica
smurilloleon@gmail.com

\begin{abstract}
Resumen
El siguiente ensayo expone el trabajo colaborativo desarrollado por el equipo del proyecto Perfiles, dinámicas y desafios de la educación costarricense: V etapa, como una buena práctica que merece ser divulgada en el Centro de Investigación en Docencia y Educación (CIDE), al cual se adscribe este proyecto. Así, recorriendo el trabajo
\end{abstract}

Recibido: 31 de marzo de 2014 - Aprobado: 18 de agosto de 2014

1 Académica del Instituto de Estudios Interdisciplinarios de la Niñez y la Adolescencia. Licenciada en Psicología y en Docencia (Universidad Estatal a Distancia).

2 Docente e investigadora en la División de Educación Rural (CIDE) en la Universidad Nacional. Filóloga hispánica y pedagoga (Universidad de Costa Rica y Universidad Estatal a Distancia). Doctora en Tecnología Educativa (Universidad de las Islas Baleares, España).

3 Profesora universitaria en la División de Educación Básica (CIDE). Máster en Artes en nseñanza de idiomas internacionales. Obtuvo un certificado de posgrado en enseñanza del inglés para personas que hablan otros idiomas (TESOL) de la California State University, Chico, California, Estados Unidos. 
que se construye entre 2010 y 2013, se concluye que el trabajo colaborativo en Perfiles, ha sido un éxito, que rebasa significativamente no solo las formas convencionales de consolidar la vida académica, sino las dinámicas que usualmente priman en el Centro para la conformación de equipos y sus formas de trabajo y comunicación.

Palabras clave: buena práctica, trabajo colaborativo, innovación, afectividad

\begin{abstract}
The following essay describes the collaborative work done by the V stage team of the project "Perfiles, dinámicas y desafios de la educación costarricense" as a good practice that should be disclosed at "Centro de Investigación en Docencia y Educación (CIDE)" to which this project is affiliated. Thus, going through the work done between 2010 and 2013, it can be concluded that the collaborative done in "Perfiles" has been successful, which significantly exceeds not only the conventional ways of consolidating academic life, but also the dynamics that usually prevent making teams and their ways of working and communicating at CIDE.
\end{abstract}

Keywords: good practice, collaborative work, innovation, affectivity

\title{
¿Qué es Perfiles y qué entendemos por buena práctica?
}

E 1 proyecto Perfiles, dinámicas y desafios de la educación costarricense, nace en el año 2004 como una propuesta del Centro de Investigación en Docencia y Educación (CIDE) de la Universidad Nacional, en busca de constituir espacios de diálogo e investigación rigurosa donde todas las unidades académicas de este Centro tuvieran parte, y donde, desde luego, se diera respuesta a necesidades y retos que el país enfrentaba en materia de educación.

En este camino, se han constituido cinco diferentes equipos de trabajo que han abordado las distintas temáticas que cada etapa del proyecto ha desarrollado. Los equipos, desde su inicio, han estado integrados por representantes académicos de las unidades del CIDE: División de 
Educación Básica (ente encargado de formar futuros profesores de I y II ciclos, inglés para niveles de primaria, educación preescolar y educación especial), División de Educación Rural (encargada de la formación de docentes especializados en educación rural para I y II ciclos y para el trabajo comunitario rural e indígena), División de Educación para el Trabajo (encargada de formar orientadores y directores educativos), División de Educología (ente encargado de formar docentes en diferentes áreas de especialización para secundaria), Instituto de Estudios Interdisciplinarios de la Niñez y la Adolescencia (ente encargado de investigaciones y proyectos de extensión sobre temas de derechos y estado de la niñez y la adolescencia). Esta conformación permite que los miembros del equipo no solo compartan conocimientos y experiencias de sus campos de especialización, sino que aprendan de sus diferentes culturas de trabajo.

$\mathrm{Al}$ finalizar la $\mathrm{V}$ fase de este proceso, y ya a las puertas de una sexta etapa -ahora como programa-, creemos que uno de los valores agregados más sobresalientes del trabajo de Perfiles es precisamente la capacidad de colaborar interdisciplinariamente y desde unidades académicas diversas, de forma tal que el trabajo colaborativo - no solo se adscribe a aspectos académicos y administrativos, sino a áreas afectivas y motivacionales; se ha constituido en una buena práctica que creemos oportuno socializar.

Para empezar, es necesario reflexionar alrededor del concepto de buena práctica. Sobre el particular, la Organización de las Naciones Unidas para la Educación, la Ciencia y la Cultura (UNESCO), señala las condiciones que definen una buena práctica:

Innovadoras: desarrollan soluciones nuevas o creativas.

Efectivas: demuestran un impacto positivo y tangible sobre la mejora. Sostenibles (...) pueden mantenerse en el tiempo y producir efectos duraderos.

Replicables: sirven como modelo para desarrollar políticas, iniciativas y actuaciones en otros lugares.

Así, una buena práctica podría ser desde una iniciativa hasta un modelo exitoso que mejore cierto proceso y, que por su difusión, posibilite aprender de los demás, construir puentes entre soluciones empíricas y la investigación, y proporcionar orientaciones para el desarrollo de otras iniciativas (Consejo Escolar, 2012). 
La buena práctica no solo permite mejorar las acciones propias, sino compartir los aprendizajes derivados de la experiencia. No obstante, la buena práctica por sí sola no genera resultados efectivos, reflexivos y analíticos; para ello se requiere de todo un procesamiento de la experiencia, dicho de otra forma, en su sistematización. Sistematizar una experiencia implica una comprensión acerca del porqué esta se está dando y por qué se desarrolla de cuál o tal manera. Necesita también un entendimiento e interpretación crítica de lo acontecido a partir de la reconstrucción del proceso (Jara, s.f.). Esta metodología representa una oportunidad para romper con las pautas convencionales acerca de la validación del conocimiento. Jara manifiesta, (s.f., p.4):

...sufrimos un paradigma de ciencia que no valora el conocimiento empírico, el saber local, y solo pone énfasis en el conocimiento acumulado, en el saber constituido académicamente. Es un error. La sistematización de experiencias es una gran posibilidad para que se expresen y se desarrollen y divulguen los conocimientos y saberes locales, que tienen mucho que aportar al enriquecimiento del pensamiento científico. Esto también permitirá asumir más creativamente nuestros proyectos, pues no tendremos una actitud pasiva que se reduce a aplicar los conocimientos y verdades que ya existen, sino que impulsaremos nuestra capacidad de crear conocimientos adecuados a la realidad que vivimos y sus particularidades.

Desde esta posición, la sistematización de buenas prácticas constituiría una metodología valiosa para la generación de conocimientos con y desde las necesidades y realidades de una población. La buena práctica es relevante especialmente para quienes la desarrollaron y fueron favorecidos por esta; pero se trata de un proceso que dejó enseñanzas para otros. Para definir qué se considera significativo en una práctica, podemos referir los principios expuestos por Rosales, Melendreras y Martínez (2008, pp.3-4), quienes refieren factores como:

Liderazgo claro -con rasgos emprendedores y participativos- por parte de (...) quienes llevan a cabo la experiencia.

Existencia de equipos de trabajo motivados y comprometidos... Apertura a otros grupos o instituciones presentes en el territorio... 
Generación de un ambiente propicio para el cambio... Es común observar el desarrollo de una cultura de colaboración y confianza entre los actores involucrados...

Visto lo anterior, debemos entonces profundizar cómo es el trabajo colaborativo e interdisciplinario puede considerarse como una buena práctica factible de sistematización y replicación.

En relación con el trabajo colaborativo, es importante acotar que este tiene como característica esencial el desarrollo de relaciones simétricas desde el conocimiento, la acción y el estatus. Los participantes de la experiencia tienen el mismo rango, un nivel similar en cuanto a conocimientos, experticias o habilidades apreciables por el grupo, y una cuestión significativa: todos gozan del mismo estatus. Esto quiere decir que las decisiones se toman de forma concensuada y que las perspectivas y criterios de todas y todos son considerados como valiosos, lo cual se refleja en la práctica, tanto en la aceptación y realimentación dada a estos como en su integración a las decisiones y productos que el equipo genere. Quienes trabajan colaborativamente comparten también un conjunto de objetivos y están interesados en su logro mediante la combinación de acciones de los participantes (Maldonado, Lizcano, Pineda, Uribe y Sequeda, 2008).

Diversos autores exponen criterios relativos a lo que caracteriza el trabajo colaborativo. En este sentido, destacan las posiciones de Barkley, Cross y Major (2007), quienes señalan:

1. En el trabajo colaborativo se estructuran actividades con una intención en particular y se privilegia el desarrollo de habilidades sociales como aceptar y pedir ayuda, la escucha y el respeto mutuos, la corresponsabilidad, la discusión y habilidades generales como la administración del tiempo, la administración de proyectos, el análisis, la síntesis, la evaluación, la toma de decisiones y la comunicación oral y escrita.

2. Cada integrante debe adquirir un compromiso evidente para trabajar en conjunto y lograr los objetivos propuestos. El trabajo es equitativo y reconocido de acuerdo con las capacidades, posibilidades y perfil de cada involucrado.

3. Al desempeñar una tarea colaborativa, los participantes deben incrementar sus conocimientos o profundizar en cuanto a la 
comprensión de ciertos tópicos. Las diferentes tareas deben buscar el cumplimiento de los objetivos, pues el trabajo efectuado pierde sentido si estos no se alcanzan.

La teoría anteriormente referida, deja claro que para que el trabajo colaborativo sea exitoso, es primordial que cada miembro del equipo reconozca no solo el trabajo y los aportes del resto, sino que se debe ser también consciente de las diferencias que se reportan de la diversidad de especialidades, experiencias, condiciones y capacidades de los participantes involucrados. Es decir, tampoco se espera que todos trabajen con la misma medida -cuantitativamente hablando-, pero sí que el trabajo desempeñado sea equitativo.

\section{La afectividad y motivación como aliciente: Un ingrediente para el éxito del trabajo colaborativo}

Dijimos párrafos atrás, que el trabajo desarrollado por el equipo que nos ocupa en este texto era valioso, no solo en el tanto se ha desarrollado una práctica académica respetuosa y colaborativa, sino porque paralelamente se han tejido relaciones afectivas y sociales que se vinculan también con un clima de trabajo gratificante, lo que sin duda repercute en la motivación y el estímulo hacia el trabajo y el equipo mismo.

Pérez, Subirá y Guitert (2008), afirman que el “...papel fundamental y básico que desempeñan los aspectos sociales, informales o afectivos del aprendizaje en el desarrollo de proyectos colaborativos con éxito" (p. 2). Así, se reflejan los elementos que han destacado en el trabajo colaborativo desarrollado por el proyecto de Perfiles. En primer lugar se enfatiza en los aspectos sociales y afectivos como ingrediente de la labor desarrollada, y como segundo punto, se valora el aprendizaje, ya que el aprender de la experticia del otro, ha sido un aspecto clave dentro del proyecto y de toda la tarea colaborativa.

La afectividad y la motivación emergen de un clima gratificante en el equipo de trabajo, siendo acertada la analogía que realizan González-Roma y Silla (1999), quienes indican que por su significado en el ámbito meteorológico, el término clima es utilizado para referirse a características del contexto psicosocial, describiendo dichas características como unidades diversas, como es el caso de los equipos de trabajo. Para estos autores, el clima laboral es un factor que incide en 
los resultados del equipo y la experiencia de sus integrantes. Cuando se potencian efectos positivos en el clima de un equipo, este influencia los demás elementos relevantes para sus miembros.

Gil, Rico y Sánchez-Manzanares (2008), indican que dentro de las dinámicas de trabajo de los equipos para cumplir las tareas asignadas, existen procesos afectivos y motivacionales entre los que destacan la cohesión, la eficacia colectiva, los procesos emocionales y el conflicto.

Estos autores plantean que "...la cohesión es la tendencia o el deseo de los miembros de un equipo de estar unidos para conseguir las metas comunes" (p.28) Lo anterior, cobra vital importancia para el desarrollo de trabajos colaborativos como el concerniente en este ensayo, pues la cohesión de los miembros se asocia de manera positiva con la eficacia, primordialmente en tareas que requieren interdependencia y coordinación, como las que ocupan al equipo Perfiles en sus tareas cotidianas.

La satisfacción y motivación son factores clave para el trabajo colaborativo desarrollado diariamente, que como bien se ha indicado, constituyen un aliciente para los miembros de este equipo y permiten continuar la tarea a pesar de altibajos o situaciones de tensión. Autores como Gil, Rico y Sánchez-Manzanares (2008), explican cómo en los equipos de trabajo es posible que se desarrollen sentimientos, emociones y estados de ánimo comunes en los miembros, gracias a las experiencias que comparten y la interacción que se da a través de las tareas atinentes a la consecución de objetivos comunes.

Ahora bien, como parte de los sentimientos y emociones que surgen de la interacción y coordinación propias de la consecución de los objetivos, es innegable que en todo equipo de trabajo existe la presencia de conflictos. Estos, usualmente, se interpretan como algo negativo -ya que pueden tener efectos contraproducentes a la eficacia del equipo, interfiriendo con el rendimiento, satisfacción, motivación y emociones de los miembros-. Los efectos de los conflictos pueden variar dependiendo de factores como la confianza, la potencia del equipo y el tipo de conflicto, ya que no es lo mismo un conflicto por incompatibilidades personales -que suele estar acompañado de afectos negativos- que un conflicto que surge de la tarea propiamente dicha, asociado a diferencias de puntos de vista.

En ambos casos, lo que la experiencia particular dicta es que, con la meta de salir adelante, la perseverancia, la comunicación y el profesionalismo, se logra avanzar y subsanar los conflictos de una u otra manera. 


\section{¿Por qué es el trabajo desarrollado por el equipo Perfiles una buena práctica?}

En primer lugar es importante recalcar que el equipo Perfiles ha logrado consolidarse como equipo de trabajo; hecho que se evidencia por la generación conjunta de todas las producciones, la realimentación franca y directa al trabajo cotidiano, la toma de decisiones concensuada, la valoración de las capacidades individuales y las relaciones construidas más allá de la obligatoriedad del vínculo laboral (afectivas, personales). Lo anterior no es sencillo, y no se logra con solo colocar un grupo de personas ante una meta en concreto: trasciende mucho esta acción, ya que los miembros deben tener

...un cierto grado de interdependencia tanto en términos de objetivos como de tarea, gestionan y mantienen sus límites y están inmersos en un contexto organizacional que limita su actividad e influye sobre sus intercambios con otros equipos dentro de la organización... (Rico, de la Hera y Tabernero, 2010 p. 48).

El hecho de que los miembros del equipo Perfiles hayan conseguido una dependencia recíproca necesaria dentro del contexto de trabajo del CIDE, ha sido indispensable para que el trabajo desarrollado sea considerado una buena práctica, pues ha logrado ser un proyecto eficaz, con logros positivos y proactivos; debido a que se ha dado una interdependencia que permite a los miembros interactuar y depender de los demás para lograr sus objetivos -representar a su unidad académica dentro del equipo- sin perder los objetivos comunes del equipo. Dicha relación se evidencia en prácticas que los miembros del equipo han interiorizado y vuelto cotidianas, por ejemplo, el trabajo por pares y luego socializado y realimentado por los demás, la construcción colectiva de textos (usando herramientas digitales como Google Drive), la discusión y toma de decisiones en conjunto, la delegación de responsabilidades en función de capacidades y condiciones de cada miembro, las reuniones vía chat o vídeo conferencia cuando deben tratarse o consultarse asuntos fuera de los días de reunión, entre otras.

El logro de una interdependencia "saludable" muestra efectos moduladores positivos dentro del desempeño del proyecto, ya que influye en procesos como las conductas de ayuda, la confianza, la comunicación, el conflicto o la flexibilidad (Rico, de la Hera y Tabernero, 2010). 
La interdependencia positiva también puede relacionarse con variables como la virtualidad y la autonomía. La virtualidad es un elemento de gran importancia para analizar el trabajo desarrollado por el equipo Perfiles como una buena práctica. Esta es definida de este modo por Rico de la Hera y Tabernero (2010):

...a partir de tres dimensiones: dependencia de los miembros respecto de las tecnologías de la información y comunicación para coordinarse y ejecutar los procesos del equipo, tipos de información que aportan las tecnologías y sincronía en las comunicaciones entre los miembros... (p. 50).

Así, se ha logrado equilibrar este elemento para aprovechar el uso de las tecnologías y el contacto cara a cara de acuerdo con el trabajo en desarrollo y beneficiando la eficacia del equipo, ya que cuando se está atendiendo alguna tarea que requiere mucha atención, interdependencia y coordinación del equipo, los niveles de virtualidad son bajos, a diferencia de cuando se realiza trabajo virtual.

Con respecto a la autonomía, Rico et al. (2010) indican que “... los efectos positivos de la autonomía sobre el rendimiento de equipo sólo se producen bajo condiciones de alta interdependencia" (p. 50), debido a que la autonomía puede permitir al equipo tomar decisiones sobre el trabajo, como los roles, horarios y otros. En el caso particular que nos ocupa, el equipo Perfiles cuenta con una alta autonomía, lo cual permite que en conjunto se puedan tomar variedad de decisiones que repercuten directamente en el rendimiento y eficacia del equipo.

De igual forma, en el desarrollo de la $\mathrm{V}$ fase del proyecto, han mediado procesos que han orientado la acción y a la conducta grupal, y que han dado su aporte para su consolidación como una buena práctica.

Uno de estos procesos medulares es la comunicación; Gil et al. (2008), explican que “...es un proceso básico en un equipo por cuanto permite el intercambio de información entre sus miembros" (p. 29), y que de esta dependen otros procesos del equipo, como la toma de decisiones y las relaciones interpersonales. En la experiencia de Perfiles, este es un elemento obligatorio, ya que se deben tomar múltiples decisiones en conjunto, debido a la alta autonomía del equipo. Además, ha sido un elemento fundamental en la resolución de conflictos, la cohesión del equipo y el logro de una interdependencia exitosa. 
Otros elementos presentes en el trabajo colaborativo realizado por este proyecto son la cooperación y la coordinación de los miembros, hechos relevantes dado que han permitido que los miembros del equipo contribuyan de manera libre y voluntaria en las tareas comunes, sincronizando los esfuerzos de todos para la meta colectiva y realizando los ajustes necesarios para ello, tal como lo exponen, Gil et al. (2008), cuando indican que la coordinación se da hasta de manera implícita en los equipos de trabajo, donde "...los miembros del equipo anticipan las acciones y necesidades de los compañeros y las demandas de la tarea, y en función de ello ajustan su propia conducta, sin necesidad de planificación o comunicación explícita entre ellos" (p. 29).

Cuanto más tiempo trabajan juntos los miembros de un equipo, mejor se conocen las habilidades de cada uno y así, mejor se coordinan sus acciones. Un ejemplo de lo anterior es que al trabajar como equipo, los académicos han logrado tener ideas innovadoras, efectivas, sostenibles y replicables, señaladas estas como buenas prácticas por la UNESCO; ya que su participación interdisciplinaria propone retos para innovar, reconociendo innovación como aquellas acciones creativas-empíricas que logran solucionar de manera efectiva y novedosa algún problema determinado.

Por ello, al ser un conjunto de personas que ha trabajado por tres años, se ha expuesto a variedad de problemas y retos que como equipo debieron conocerse y solventar eficazmente. Como ejemplo de lo anterior, se tiene la obtención de un laboratorio de computadoras móvil se convierte en un logro del equipo, al participar para el proyecto FID Holanda CECC-SICA (Coordinación Educativa y Cultural Centroamericana/ Sistema de la Integración Centroamericana) 2010-2013, porque se capacita a un grupo de académicos del CIDE con el fin de promover la enseñanza con el uso de Tecnologías de la Información y Comunicación, desde los académicos. Esta actividad no solamente enriquece el quehacer del Centro sino que también se analiza como una experiencia, en donde se produce como fruto compartir el laboratorio con profesores y estudiantes: a partir de una experiencia relevante para el equipo se favorece a otros, a la institución y al país.

A través de la realización de las metas y objetivos fijados por el equipo Perfiles, se han combinado los recursos personales de cada uno de los miembros del equipo, poniendo a disposición de las metas comunes, las habilidades, conocimientos y destrezas personales. De este modo, como equipo de trabajo se han condensado los afectos y emociones de los miembros, creando una fusión que, de manera innegable, 
incide en el desempeño del equipo en general, dando como resultado un ambiente propicio y gratificante. No sólo de manera social, sino desde un punto de vista más constructivo de la labor del Proyecto Perfiles, el ambiente y la generación de investigaciones hace que este grupo plasme ideas o proyectos que puedan seguir desarrollándose con el tiempo. Cómo último punto, es significativo señalar que uno de los elementos que han marcado a Perfiles, ha sido su capacidad de adaptarse a los cambios en la composición del equipo. Rico et al. (2010) indican que si no se dan de esta manera, los cambios pueden perjudicar la eficacia de los equipos, afectando el rendimiento y la coordinación. En nuestro caso, si bien los cambios han sido muchos, la gradualidad de éstos y la apertura y actitud del resto de los miembros han facilitado la transición, y lejos de perjudicar el desempeño global, ha permitido fortalecer capacidades y aprovechar las individualidades y experticias que han pasado por el grupo.

\section{Referencias bibliográficas}

Barkley, E, Cross, K. y Major, C. (2007). Técnicas de aprendizaje colaborativo. Madrid: Ediciones Morata.

Coll, C y Monereo, C (2008) Psicología de educación virtual. Madrid: Editorial Morata. Consejo Escolar de Madrid (2012) Sobre el concepto de buena práctica. Recuperado de_http://www.igualdadycalidadcba.gov.ar/SIPEC-CBA/Capacitacion2012/ DocumentosSecundaria/Preceptores/Sobre $\% 20 \mathrm{el} \% 20$ concepto $\% 20 \mathrm{de} \% 20$ Buena\%20Practica.pdf

Gil, F., Rico, R. y Sánchez-Manzanares, M. (2008). Eficacia de equipos de trabajo. Papeles del Psicólogo, 29(1), 25-31.

González-Romá, V. y Silla, J. M. P. (1999). Clima en las organizaciones laborales y en los equipos de trabajo. Revista de psicología general y aplicada: Revista de la Federación Española de Asociaciones de Psicología, 52(2), 269-285.

Jara, O. (s.f.) El desafio político de aprender de nuestras prácticas. San José: Publicaciones Alforja. Recuperado de http://www.cepalforja.org/sistem/documentos/ aprenderdepracticas.pdf

Maldonado, L, Lizcano, A. R., Pineda, E., Uribe, V. y Sequeda, J. (2008). Comunidades de aprendizaje mediadas por redes informáticas. Educación y Educadores, 11(1) pp. 199-224.

Pérez, M., Subirá, M. y Guitert Catasús, M. (2008). La dimensión social del aprendizaje colaborativo virtual. Revista de educación a distancia, 18, 1-21.

Rico, R., de la Hera, C. M. A. y Tabernero, C. (2010). Efectividad de los Equipos de Trabajo, una Revisión de la Última Década de Investigación (1999-2009). Revista de Psicología del Trabajo y de las Organizaciones, 26(1), 47-71.

Rosales, M., Melendreras, I y Martínez, M (2008) Guía metodológica para la sistematización de buenas prácticas. Programa de descentralización y fortalecimiento municipal: Ciudad Guatemala. 